\title{
HARMONIC NULL SETS AND THE PAINLEVÉ THEOREM
}

\author{
J. L. SCHIFF
}

\begin{abstract}
A less restrictive condition on an open Riemann surface than has been formerly known for a subset of the ideal boundary of a resolutive compactification to have harmonic measure zero is demonstrated. Then a generalized version of a classical theorem of Painlevé is established in this framework.
\end{abstract}

Recently, Arsove and Leutwiler [2] proved a generalization of the classical theorem of Painlevé which states that an analytic function on a Jordan region which tends to zero at each point of a nondegenerate boundary arc vanishes identically. Their result stemmed from an important new characterization of harmonic null sets. It is well known [1] that the existence of a positive harmonic function on a bounded region which tends to $\infty$ on a boundary set $E$ is necessary and sufficient for $E$ to have harmonic measure zero. In [2], the requirement of positiveness is disposed of.

A similar situation has existed in the field of potential theory on Riemann surfaces. The existence of a positive superharmonic function on $R$ whose lim inf tends to $\infty$ at each point of a subset of a suitable ideal boundary, has long been accepted as a sufficient condition for the subset to have harmonic measure zero. However, it can be demonstrated that here also positiveness is not required, and Painlevé's theorem can be extended to Riemann surfaces as well.

THEOREM. Let $R^{*}$ be a resolutive compactification of an open Riemann surface $R$, and $\Delta=R^{*}-R$. If a superharmonic function $s$ on $R$ tends to $\infty$ at all points of $E \subset \Delta$, then the harmonic measure $\omega$ of $E$ is zero.

Proof. Let $G=\{z \in R \mid s(z)>0\}$. Then $G$ is open in $R$, and set

$$
A=\left\{\zeta \in \Delta-\operatorname{cl}(R-G) \mid \liminf _{z \rightarrow \zeta} s(z)=\infty\right\} .
$$

Received by the editors July 31, 1973.

AMS (MOS) subject classifications (1970). Primary 31A20; Secondary 30A50.

(C) American Mathematical Society 1974 
By Hilfssatz 8.8 of [3], $\omega(A)=0$. For any $\zeta \in E$, if $\zeta \notin A$, then we must have $\zeta \in \operatorname{cl}(R-G)$ since $\lim _{z \rightarrow \zeta} s(z)=\infty$. Hence there exists a net $\left\{z_{\alpha}\right\} \subset$ $R-G$ such that $z_{\alpha} \rightarrow \zeta$. But $\zeta \in E$ implies that $s\left(z_{\alpha}\right) \rightarrow \infty$, which contradicts the fact that $s\left(z_{\alpha}\right) \leqq 0$. It follows that $E \subset A$ and $\omega(E)=0$ as desired.

The theorem is also valid in the theory of harmonic spaces.

Painleve's classical result can now be generalized to the following:

COROLlary. Let $f$ be analytic on $R, f \rightarrow 0$ at all points of $E \subset \Delta$, where $\omega(E)>0$. Then $f \equiv 0$.

Proof. If $f \not \equiv 0$, let $u=-\log |f|$. Then $u$ is superharmonic on $R$, and $u \rightarrow \infty$ at all points of $E$. Thus $\omega(E)=0$, a contradiction.

\section{BIBLIOGRAPHY}

1. M. Arsove, The Wiener-Dirichlet problem and the theorem of Evans, Math. Z. 103 (1968), 184-194. MR 36 \#4009.

2. M. Arsove and H. Leutwiler, Painlevé's theorem and the Phragmén-Lindelöf maximum principle, Math. Z. 122 (1971), 227-236.

3. C. Constantinescu and A. Cornea, Ideale Ränder Riemannscher Flächen, Ergebnisse der Math. und ihrer Grenzgebiete, Band 32, Springer-Verlag, Berlin, 1963, 244 pp. MR 28 \#3151.

4. P. Painlevé, Sur les lignes singulières des fonctions analytiques, Ann. de Toulouse 2 (1888), 1-130.

Department of Mathematics, University of Auckland, Auckland, New ZEALAND 